\title{
Potenciais fatores associados a maior chance de recidiva de erisipela
}

Potential factors associated with increased chance of erysipelas recurrence Posibles factores asociados a mayor probabilidad de recidiva de erisipela

\author{
Etiene Souza Madeira ${ }^{1}$ ib htps://orcid.org/0000-0001-9012-6480 \\ Leila Neto Figueredo ${ }^{1}$ (1) https://orcid.org/0000-0002-5253-3912 \\ Bruna Maiara Ferreira Barreto Pires² ${ }^{2}$ nttps:///orcid.org/0000-0002-5584-8194 \\ Sônia Regina de Souza ${ }^{1}$ ic https://orcid.org/0000-0001-7981-0038 \\ Priscilla Alfradique de Souza' io https://orcid.org/0000-0002-4625-7552
}

$\begin{aligned} \text { Como citar: } & \\ \text { Madeira ES, Figuredo } & \end{aligned}$

Madeira ES, Figueredo LN, Pires BM, Souza SR,

Souza PA. Potenciais fatores associados a maior chance de recidiva de erisipela. Acta Paul Enferm.

DOI

http://dx.doi.org/10.37689/acta-ape/2022A002822

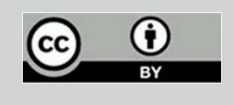

Descritores

Erisipela; Saúde do idoso; Ferimentos e lesões; Recidiva

Keywords

ds and injuries;

Recurrence

Descriptores

Erisipela; Salud del anciano; Heridas y traumatismos; Recurrencia

Submetido

28 de Setembro de 2020

Aceito

26 de Maio de 2021

\section{Autor correspondente}

Etiene Souza Madeira

Email: souza.etiene@gmail.com

Editor Associado (Avaliação pelos pares): Camila Takao Lopes (https://orcid.org/0000-0002-6243-6497) Escola Paulista de Enfermagem, Universidade Federal de São Paulo, São Paulo, SP, Brasil

\section{Resumo}

Objetivo: Analisar os fatores relacionados à recidiva de erisipela em adultos e idosos.

Métodos: Estudo de coorte retrospectivo com 235 adultos e idosos admitidos em um hospital com diagnóstico de erisipela entre 2012 e 2019. Investigaram-se fatores sociodemográficos e clínicos relacionados a maior chance de recidiva de erisipela no período por meio de análises uni e bivariada, com $p<0,05$ considerado significativo.

Resultados: A prevalência de recidiva de erisipela foi de $25,5 \%(n=60)$. Os fatores significativamente associados à recidiva foram insuficiência venosa $(\mathrm{p}=0,002 ; \mathrm{OR}=2,597 ; \mathrm{IC}=1,4-4,7)$ e uso de penicilina $(\mathrm{p}<$ 0,000; $\mathrm{OR}=7,042 ; \mathrm{IC}=2,5-19,7)$.

Conclusão: a insuficiência venosa se associa a chance duas vezes maior de recidiva de erisipela e 0 uso de penicilina se associa a risco sete vezes maior para sua recidiva.

\section{Abstract}

Objective: To analyze factors related to erysipelas recurrence in adults and older adults.

Methods: Retrospective cohort study with 235 adults and older adults admitted to a hospital diagnosed with erysipelas between 2012 and 2019. Sociodemographic and clinical factors related to a greater chance of erysipelas recurrence in the period were investigated through uni and bivariate analyses, with $p<0.05$ considered significant.

Results: The prevalence of erysipelas recurrence was $25.5 \%(n=60)$. Factors significantly associated with recurrence were venous insufficiency $(\mathrm{p}=0.002 ; \mathrm{OR}=2.597 ; 95 \% \mathrm{Cl}=1.4-4.7)$ and use of penicillin $(\mathrm{p}<$ $0.000 ; \mathrm{OR}=7.042 ; 95 \% \mathrm{Cl}=2.5-19.7)$.

Conclusion: venous insufficiency is associated with a twice greater chance of erysipelas recurrence and the use of penicillin is associated with a seven times greater risk for its recurrence.

\section{Resumen}

Objetivo: Analizar los factores relacionados con la recidiva de erisipela en adultos y adultos mayores.

Métodos: Estudio de cohorte retrospectivo con 235 adultos y adultos mayores ingresados en un hospital con diagnóstico de erisipela entre 2012 y 2019. Se investigaron factores sociodemográficos y clínicos relacionados con una mayor probabilidad de recidiva de erisipela en el período mediante análisis uni y bivariados, con $p<0,05$ considerado significativo. 
Resultados: La prevalencia de recidiva de erisipela fue del $25,5 \%(n=60)$. Los factores significativamente asociados con la recidiva fueron insuficiencia venosa $(p=0,002 ; 0 R=2,597 ; I C=1,4-4,7)$ y uso de penicilina $(p<0,000 ; 0 R=7,042 ; I C=2,5-19,7)$.

Conclusión: La insuficiencia venosa está relacionada con una probabilidad dos veces mayor de recidiva de erisipela, y el uso de penicilina está relacionado con un riesgo siete veces mayor de recidiva.

\section{Introdução}

A recidiva de erisipela acarreta em repercussóes desfavoráveis devido as internaçóes recorrentes, antibioticoterapia prolongada e comprometimento cutâneo. Os pacientes acometidos em sua maioria são idosos com comorbidades, nos quais a recorrência da erisipela é agravada por doenças pré-existentes. Para pacientes com problemas como insuficiência venosa, devido ao comprometimento do retorno venoso, essas feridas podem acarretar perda de grande extensão de tecido e complicaçóes graves, levando a necessidade de abordagem cirúrgica e até, em alguns casos, amputação do membro afetado.

A erisipela é uma infecção de pele, com intenso comprometimento cutâneo que geralmente está associado a pacientes que possuem outras patologias. $(1,2)$ Essa infecção cutânea é geralmente causada pela a bactéria estreptococo beta-hemolítico do grupo A, o Streptococcus pyogenes, mas também pode ocorrer por outras bactérias em menor incidência. ${ }^{(2)}$

A erisipela acontece por meio de uma porta de entrada, normalmente uma fissura na pele, que pode ocorrer por diversos fatores, como, trauma, micose, úlcera, no qual o agente bacteriano entra, afetando o tecido cutâneo e o sistema linfático. Os fatores de risco mais comumente observados são idade avançada, sexo masculino, cirurgia venosa prévia, obesidade, uso crônico de drogas lícitas, linfedema, diabetes, imunidade baixa e insuficiência venosa. ${ }^{(3)}$

A enfermagem tem papel fundamental no cuidado ao paciente com feridas decorrentes de erisipela, pois, além de identificar aspectos que influenciam o acometimento, são responsáveis por avaliar, prescrever e implementar o cuidado, promovendo atendimento integral. Ressalta-se, ainda, que a assistência de enfermagem à pessoa com feridas está implícita nas atribuiçóes do enfermeiro. ${ }^{(4)}$

Cabe destacar que o principal tratamento farmacológico para erisipela é a antibioticoterapia. De acordo com o microrganismo envolvido, a penicilina é o antibiótico de referência, mas também po- dem ser utilizados outros antibióticos, como, clindamicina, claritromicina, cefalexina, flucloxacilina, moxifloxacina, entre outros. ${ }^{(3)}$

Complicações devido a erisipela são raras, mas podem ocorrer principalmente nos casos não tratados em tempo hábil, dentre os quais ulceraçóes superficiais e profundas, linfedema, abcessos e trombose de veias. ${ }^{(5)}$

Deste modo, torna-se importante a equipe de saúde compreender os padróes comportamentais da doença, ou seja, público afetado com a doença, seu curso e desfecho. Isto se aplica aos pacientes acometidos por recidiva de erisipela, principalmente por ser uma afecção clínica encontrada tanto ambulatorialmente, como também nas clínicas médica, cirúrgica e setores de alta complexidade. Nesse sentido, identificar fatores de risco a recidiva de erisipela, além de permitir uma assistência preventiva, possibilitará direcionar o melhor tratamento para a mesma, uma vez que o antibiótico mais usado hoje para essa patologia é a penicilina, porém muitos estudos vem questionando seu uso. Dessa forma, há contrapontos a serem melhor discutidos acerca da erisipela, justificando esse estudo.

Além disso, revisão integrativa realizada em 2016 aponta a necessidade de mais estudos sobre a temática. ${ }^{(6)}$ Ademais, são escassos os estudos sobre recidiva de erisipela no Brasil, o que causa grande impacto na saúde dos pacientes acometidos com comorbidades prévias, principalmente idosos. Estudo de maior relevância encontrado sobre recidiva de erisipela, especificamente, foi na Suécia no Departamento de Doenças Infecciosas do Hospital Universitário Skåne publicado em 2014, com 502 pacientes dos quais $145(28,9 \%)$ apresentaram recidiva. $^{(1)}$

Para ratificar os aspectos supracitados, ao pesquisarmos o descritor erisipela (erysipelas) na base de dados MEDLINE via Pubmed são encontrados nos últimos 5 anos 317 estudos, ao cruzar o termo erisipela (erysipelas) e recidiva (relapse) são encontrados apenas 32 artigos, dentre os quais 4 abordam 
questóes relacionadas sobre recidiva de erisipela e somente 1 destaca a associação de fatores clínicos com a recidiva de erisipela. A maioria dos estudos encontrados descrevem as características clínicas dos pacientes com recidiva, sem fazer associaçóes ou apenas apontam tratamentos, principais causas e microrganismos de forma descritiva, justificando, assim, a realização desse estudo.

Portanto, a partir desses dados, este estudo tem como objetivo analisar os fatores de risco relacionados a recidiva de erisipela em adultos e idosos.

\section{Métodos}

Trata-se de estudo de coorte retrospectivo com 235 pacientes que possuíam o diagnóstico de erisipela CID A 46. A coleta foi realizada através de prontuário eletrônico individual (PEI) dos pacientes. O PEI contém informaçóes cadastrais, evoluçôes de toda a equipe de saúde sobre o atendimento aos pacientes, as prescriçóes medicamentosas e os ambulatórios em que o paciente fora atendido.

Os critérios de inclusão foram adultos (18 a 59 anos) e idosos (60 anos ou mais), com o diagnóstico de erisipela, atendidos no hospital, que possuíam registro no PEI dos pacientes e tinham o diagnóstico: CID A46 - Erisipela. Os critérios de exclusão foram prontuários que não continham informações suficientes.

$\mathrm{O}$ instrumento de coleta de dados constituiu por dados socioeconômicos e informaçóes sobre a internação hospitalar, como risco de queda, sintomas apresentados, porta de entrada da doença, comorbidades, recidiva da doença, hábitos de vida, condições nutricionais, uso de antibioticoterapia (antibióticos usados na primeira internação por erisipela e não na recidiva) e de outras medicaçóes, exames laboratoriais e complicaçóes graves. A polifarmácia foi definida pelo uso de cinco ou mais medicações de forma contínua.

$\mathrm{O}$ estudo trabalhou com a população total( ${ }^{(7-9)} \mathrm{a}$ partir de dados referentes ao período de 01 de janeiro de 2012 a 01 de janeiro de 2019 de pacientes hospitalizados na clínica médica registrados no PEI. O início da coleta a partir do ano de 2012 foi definido em função do início dos registros de enfermagem e da clínica médica no hospital da população em estudo. Optou-se por cumprir o tamanho recomendado na literatura de pelo menos 30 casos. A literatura estatística e experimental ressalta que sempre que o tamanho de participantes for menor que 30, a análise estatística pode ser dificultada e o desempenho dos testes estatísticos pode estar comprometido. ${ }^{(10)} \mathrm{O}$ tamanho maior ou igual a 30 é considerado na estatística como grande amostra. ${ }^{(11)}$ As grandes amostras são aquelas onde se pode verificar a densidade de probabilidade de forma definida e estão amparadas pelo Teorema Central do Limite. ${ }^{(12)}$

A análise foi realizada através de planilha eletrônica do programa Microsoft Office Excel 2016 e com o auxílio do programa R 3.4.3 e do software Statistical Package for Social Sciences (SPSS) 21. Foi considerado p-valor menor que 0,05 e intervalo de confiança de $95 \%$. Foi efetuada análise univariada com a finalidade de descrever o perfil dos pacientes com erisipela que foram internados no hospital. Em seguida, fez-se a análise bivariada para analisar as relaçóes existentes entre recidiva e os seus respectivos fatores de risco. Também foi calculado o Odds Ratio (OR) para verificar as chances de um indivíduo ter recidiva com relação aos fatores de risco, e o teste do qui-quadrado para verificar a associação entre os fatores de risco com a ocorrência de recidiva.

$O$ estudo foi submetido e registrado na Plataforma Brasil sob o número de Certificado de Apresentação para Apreciação Ética (CAEE) 20106819.2.0000.5285 e aprovado pelo Comitê de Ética em Pesquisa da Universidade Federal do Estado do Rio de Janeiro sob o número 3.563.200. Foi assinado a dispensa do Termo de Consentimento Livre e Esclarecido (TCLE). O estudo atendeu às normas nacionais e internacionais de ética em pesquisa envolvendo seres humanos, de acordo com a resolução 466/12.

\section{Resultados}

No período de 2012 a 2019, identificou-se como população amostral total na clínica médica 25.952 pessoas. Para a população integrante deste estudo, 
foram identificados 235 pacientes com erisipela atendidos pela clínica médica no período referido acima. A prevalência foi do sexo masculino, com $51,1 \%$. Destes $40,1 \%$ eram idosos, e apresentavam-se na faixa etária predominantemente, de 70 a 79 anos. Os locais mais acometidos foram os membros inferiores, totalizando $97 \%$ da população geral do estudo, sendo comum o aparecimento em uma única perna (Tabela 1).

No que tange a porta de entrada para a erisipela, traumas foram prevalentes com $20,4 \%$ dos casos. Etilismo foi o hábito de vida registrado com mais frequência, 8,9\% dos casos. A polifarmácia esteve presente em 31,1\% dos casos, com média de 3 antibióticos utilizados em uma mesma internação, por aproximadamente 16,8 dias de antibioticoterapia.

A tabela 1 condensa os dados sóciodemográficos e clínicos dos adultos e idosos incluídos no estudo.

Pacientes que tiveram apenas um episódio de erisipela totalizaram $74,4 \%$ da população da pesquisa e $25,6 \%$ dos pacientes apresentaram recidiva da doença. Não foi identificada relação de recidiva de erisipela com comorbidades e idade, conforme demonstrado a seguir, destacam-se hipertensão $(\mathrm{OR}=1,496 ; \mathrm{IC}=0,7-3,1)$, obesidade $(\mathrm{OR}=1,495$; IC $0,7-2,8)$. A faixa etária revelou que idosos tem risco maior de recidiva $(\mathrm{OR}=1,194 ; \mathrm{IC}=0,5 ; 2,5)$. Esses dados estão demonstrados na tabela 2. Quanto aos hábitos de vida, também não foi identificada relação de causa e efeito. No que tange aos sinais e sintomas, não foi observada relação de risco, ou seja, febre, dor no local e edema (respectivamente $\mathrm{OR}=1,353, \mathrm{IC}=0,7-2,4 ; \mathrm{OR}=1,645, \mathrm{IC}=0,8$ $3,3)$; $\mathrm{OR}=1,399, \mathrm{IC}=0,6-3,23)$ não apresentaram relação com maior risco de recidiva de erisipela. Os dados estão descritos na tabela 3.

Pacientes que fazem uso contínuo de medicaçôes $(\mathrm{OR}=1,524 ; \mathrm{IC}=0,6-3,5)$ e polifarmácia $(\mathrm{OR}=1,714$; IC: $0,9-3,1)$ não apresentaram maior risco de recidiva. No que tange a antibioticoterapia usada na primeira internaçáo, o uso de penicilina apresentou sete vezes maior risco $(p<0,000$; OR 7,042; IC= 2,5-19,7) demonstrando a relação do uso da mesma com a recidiva de erisipela. Além disso, a insuficiência venosa apresentou risco duas vezes maior $(p=0,002 ; O R=2,597 ; \mathrm{IC}=1,4-4,7)$,
Tabela 1. Caracterização sociodemográfica e clínica dos adultos e idosos com erisipela

\begin{tabular}{|c|c|c|c|}
\hline $\begin{array}{l}\text { Perfil dos pacientes com } \\
\text { erisipela }\end{array}$ & $\begin{array}{c}\text { Total } \\
(\mathrm{n}=235) \\
\mathrm{n}(\%)\end{array}$ & $\begin{array}{c}\text { Adulto } \\
(\mathrm{n}=48) \\
\mathrm{n}(\%)\end{array}$ & $\begin{array}{c}\text { Idoso } \\
(\mathrm{n}=187) \\
\mathrm{n}(\%)\end{array}$ \\
\hline \multicolumn{4}{|l|}{ Faixa etária } \\
\hline Até 29 anos & $3(1,3)$ & $3(6,2)$ & - \\
\hline 30 a 39 anos & $4(1,7)$ & $4(8,3)$ & - \\
\hline 40 a 49 anos & $18(7,7)$ & $18(37,5)$ & - \\
\hline 50 a 59 anos & $23(9,8)$ & $23(47,9)$ & - \\
\hline 60 a 69 anos & $71(30,2)$ & - & $71(38,0)$ \\
\hline 70 a 79 anos & $75(31,9)$ & - & $75(40,1)$ \\
\hline 80 ou mais & $41(17,4)$ & - & $41(21,9)$ \\
\hline \multicolumn{4}{|l|}{ Sexo } \\
\hline Masculino & $120(51,1)$ & $24(50,0)$ & $96(51,3)$ \\
\hline Feminino & $115(48,9)$ & $24(50,0)$ & $91(48,7)$ \\
\hline \multicolumn{4}{|l|}{ Local Acometido } \\
\hline Membro inferior esquerdo & $93(39,6)$ & $23(47,9)$ & $70(37,4)$ \\
\hline Membro inferior direito & $91(38,7)$ & $20(41,7)$ & $71(38,0)$ \\
\hline Ambos membros inferiores & $44(18,7)$ & $4(8,3)$ & $40(21,4)$ \\
\hline Membro superior direito & $3(1,3)$ & $1(2,1)$ & $2(1,1)$ \\
\hline Membro superior esquerdo & $3(1,3)$ & - & $3(1,6)$ \\
\hline Ambos membros superiores & $1(0,4)$ & - & $1(0,5)$ \\
\hline \multicolumn{4}{|l|}{ Porta de entrada } \\
\hline Sem registro* & $62(26,4)$ & $14(29,2)$ & $48(25,7)$ \\
\hline Traumas & $48(20,4)$ & $9(18,8)$ & $39(20,9)$ \\
\hline Úlceras & $38(16,2)$ & $6(12,5)$ & $32(17,1)$ \\
\hline Feridas & $34(14,5)$ & $6(12,5)$ & $28(15,0)$ \\
\hline Micose & $30(12,8)$ & $9(18,8)$ & $21(11,2)$ \\
\hline Ferida operatória & $10(4,3)$ & $2(4,2)$ & $8(4,3)$ \\
\hline Picada de inseto & $5(2,1)$ & $2(4,2)$ & $3(1,6)$ \\
\hline Outras ${ }^{\star \star}$ & $8(4,3)$ & - & $8(4,3)$ \\
\hline \multicolumn{4}{|l|}{ Hábitos de vida } \\
\hline Etilista ativo & $21(8,9)$ & $4(8,3)$ & $17(9,1)$ \\
\hline Tabagista ativo & $14(6,0)$ & - & $14(7,5)$ \\
\hline \multicolumn{4}{|l|}{ Polifarmácia } \\
\hline $\operatorname{Sim}$ & $73(31,1)$ & $13(27,1)$ & $60(32,1)$ \\
\hline \multicolumn{4}{|l|}{ Número de antibióticos utilizados } \\
\hline Média (SD $\left.{ }^{\star \star \star}\right)$ & $3,0(S D=1,501)$ & $3,1(\mathrm{SD}=1,453)$ & $3,0(\mathrm{SD}=1,515)$ \\
\hline Mediana (25\%, 75\%) & $3(2 ; 4)$ & $3(2 ; 4)$ & $3(2 ; 4)$ \\
\hline \multicolumn{4}{|l|}{$\begin{array}{l}\text { Número de dias de } \\
\text { antibioticopterapia }\end{array}$} \\
\hline Média (SD) & $\begin{array}{c}16,8(\mathrm{SD}= \\
10,008)\end{array}$ & $\begin{array}{c}16,9(\mathrm{SD}= \\
9,636)\end{array}$ & $\begin{array}{c}16,8(\mathrm{SD}= \\
10,127)\end{array}$ \\
\hline Mediana $(25 \%, 75 \%)$ & $14(10 ; 20)$ & $16(10 ; 22)$ & $14(10 ; 20)$ \\
\hline
\end{tabular}

essas variáveis apresentaram significância estatística uma vez que o valor de Intervalo de Confiança náo perpassa pelo numero 1. Esses dados estâo apresentados na tabela 3 .

\section{Discussão}

Nesse estudo, a maioria dos pacientes com erisipela eram idosos, o que corrobora estudo da Suécia, que 
Tabela 2. Fatores de risco associados a recidiva de erisipela dos adultos e idosos com erisipela

\begin{tabular}{|c|c|c|c|c|c|c|}
\hline \multirow{2}{*}{ Variáveis } & \multirow{2}{*}{$\begin{array}{c}\text { Com recidiva } \\
(n=60) \\
n(\%)\end{array}$} & \multirow{2}{*}{$\begin{array}{c}\text { Sem recidiva } \\
(\mathrm{n}=175) \\
\mathrm{n}(\%)\end{array}$} & \multirow[b]{2}{*}{ Odds Ratio } & \multicolumn{2}{|c|}{ IC de $95 \%$} & \multirow[b]{2}{*}{$p$-value ${ }^{\star}$} \\
\hline & & & & Limite Inferior & Limite Superior & \\
\hline \multicolumn{7}{|l|}{ Comorbidades } \\
\hline Hipertensão arterial sistêmica & $49(81,7)$ & $131(74,9)$ & 1,496 & 0,715 & 3,129 & 0,282 \\
\hline Diabetes mellitus & $28(46,7)$ & $86(49,1)$ & 0,906 & 0,503 & 1,629 & 0,741 \\
\hline Obesidade & $18(30,0)$ & $39(22,3)$ & 1,495 & 0,775 & 2,883 & 0,229 \\
\hline Insuficiência venosa & $33(55,0)$ & $56(32,0)$ & 2,597 & 1,426 & 4,731 & 0,002 \\
\hline \multicolumn{7}{|l|}{ Sexo } \\
\hline Masculino & $30(50,0)$ & $90(51,4)$ & 0,944 & 0,525 & 1,698 & 0,849 \\
\hline Feminino & $30(50,0)$ & $85(48,6)$ & - & - & - & - \\
\hline \multicolumn{7}{|l|}{ Faixa etária } \\
\hline Idoso & $49(81,7)$ & $138(78,9)$ & 1,194 & 0,565 & 2,523 & 0,641 \\
\hline Adulto & $11(18,3)$ & $37(21,1)$ & - & - & - & - \\
\hline \multicolumn{7}{|l|}{ Hábitos de vida } \\
\hline Etilista & $5(8,3)$ & $16(9,1)$ & 0,903 & 0,316 & 2,581 & 0,850 \\
\hline Tabagista & $6(10,0)$ & $8(4,6)$ & 2,319 & 0,770 & 6,982 & 0,125 \\
\hline \multicolumn{7}{|l|}{ Faz uso de medicações } \\
\hline Sim & $51(86,4)$ & $138(80,7)$ & 1,524 & 0,660 & 3,519 & 0,321 \\
\hline \multicolumn{7}{|l|}{ Polifarmácia } \\
\hline Sim & $24(40,0)$ & $49(28,0)$ & 1,714 & 0,929 & 3,164 & 0,083 \\
\hline
\end{tabular}

${ }^{*}$ Teste Qui-quadrado

Tabela 3. Variáveis sobre o diagnóstico e tratamento da recidiva de erisipela nos adultos e idosos com erisipela

\begin{tabular}{|c|c|c|c|c|c|c|}
\hline \multirow[b]{2}{*}{ Variáveis } & \multirow{2}{*}{$\begin{array}{c}\text { Com recidiva } \\
(n=60) \\
n(\%)\end{array}$} & \multirow{2}{*}{$\begin{array}{c}\text { Sem recidiva } \\
(\mathrm{n}=175) \\
\mathrm{n}(\%)\end{array}$} & \multirow[b]{2}{*}{ Odds Ratio } & \multicolumn{2}{|c|}{ IC de $95 \%$} & \multirow[b]{2}{*}{$p$-value* } \\
\hline & & & & Limite inferior & Limite superior & \\
\hline \multicolumn{7}{|l|}{ Sinais e sintomas } \\
\hline Febre & $34(56,7)$ & $86(49,1)$ & 1,353 & 0,750 & 2,442 & 0,314 \\
\hline Dor no local & $48(80,0)$ & $124(70,9)$ & 1,645 & 0,807 & 3,352 & 0,168 \\
\hline Edema & $52(86,7)$ & $144(82,3)$ & 1,399 & 0,604 & 3,239 & 0,431 \\
\hline Hiperemia & $47(78,3)$ & $146(83,4)$ & 0,718 & 0,670 & 2,896 & 0,374 \\
\hline Bolhas & $19(31,7)$ & $76(43,4)$ & 0,604 & 0,325 & 1,123 & 0,109 \\
\hline \multicolumn{7}{|l|}{ Local acometido } \\
\hline Ambos membros inferiores & $12(20,0)$ & $32(19,0)$ & 1,063 & 0,507 & 2,228 & 0,873 \\
\hline Um membro inferior & $48(80,0)$ & $136(81,0)$ & - & - & - & - \\
\hline \multicolumn{7}{|l|}{ Número de antibióticos } \\
\hline 2 ou mais & $52(86,7)$ & $152(86,9)$ & 0,984 & 0,415 & 2,333 & 0,970 \\
\hline 1 & $8(13,3)$ & $23(13,1)$ & - & - & - & - \\
\hline \multicolumn{7}{|l|}{ Número de dias antibioticoterapia } \\
\hline 15 ou mais & $29(48,3)$ & $86(49,1)$ & 0,968 & 0,538 & 1,741 & 0,914 \\
\hline Até 14 & $31(51,7)$ & $89(50,9)$ & & & & \\
\hline \multicolumn{7}{|l|}{ Antibióticos utilizados } \\
\hline Ciprofloxacino & $14(23,3)$ & $41(23,4)$ & 0,995 & 0,497 & 1,989 & 0,998 \\
\hline Clindamicina & $23(38,3)$ & $54(30,9)$ & 1,393 & 0,756 & 2,567 & 0,287 \\
\hline Amoxicilina + clavulanato & $29(48,3)$ & $74(42,3)$ & 1,277 & 0,709 & 2,300 & 0,415 \\
\hline Moxifloxacino & $20(33,3)$ & $55(31,4)$ & 1,091 & 0,584 & 2,037 & 0,785 \\
\hline Penicilina & $12(20,0)$ & $6(3,4)$ & 7,042 & 2,511 & 19,745 & $<0,000$ \\
\hline Trimetoprima + sulfametoxazol & $18(30,0)$ & $57(32,6)$ & 0,887 & 0,470 & 1,676 & 0,712 \\
\hline
\end{tabular}

${ }^{\star}$ Teste Qui-Quadrado

apresentou a mesma prevalência. ${ }^{(13)}$ Cabe destacar que estudo na África diverge quanto a esse dado sociodemográfico pela característica de expectativa de vida nesse país, apresentando prevalência de casos de erisipela em adultos. ${ }^{(14)} \mathrm{A}$ idade avançada está diretamente relacionada a maior fragilidade e maior propensão a comorbidades que contribuem para a recidiva de erisipela. ${ }^{(15)}$ Desta forma, nesse estudo ser idoso não significou maior risco de recidiva de erisipela.

Com relação ao gênero, nesse estudo houve predominância discreta do sexo masculino, com $51,1 \%$ dos participantes. Tais evidências corroboram com dados de outros estudos que houve prevalência de 
mais de $58 \%$ de pacientes com recidiva sendo do sexo masculino. ${ }^{(1,13)}$ Contudo, estudos da Eslovênia, África, e também do Brasil tiveram prevalência do sexo feminino nos pacientes com diagnóstico de erisipela. ${ }^{(14,16,17)}$ Os estudos epidemiológicos apresentam maior frequência de doenças venosas nos membros inferiores em mulheres em comparação aos homens, principalmente relacionado a questóes hormonais femininas. ${ }^{(18)}$ Pesquisadores asseguram que o sexo feminino possui predisposição para o desenvolvimento de insuficiência venosa pela ação de estrógenos, uso de anticoncepcionais e gestação.

A localização predominante da erisipela foi observada nos membros inferiores corroborando estudos alemãs que evidenciaram que as pernas são os locais mais afetados, seguidos dos braços e face. ${ }^{(13)}$ Nos resultados desta pesquisa, a principal porta de entrada foi por meio de traumas, seguido por úlceras, feridas e micoses. Estudo no Brasil aponta que micose foi a principal porta de entrada, seguido por fissuras nos pés, úlceras em membros inferiores e intertrigo inflamatório. ${ }^{(17)}$ Apesar de micoses não serem a porta de entrada mais prevalente, temperaturas mais altas predispóem ao desenvolvimento de lesóes fúngicas na pele e aumentam a estase venosa e linfática, favorecendo as fissuras da pele como portas de entrada. ${ }^{(16)}$

Sendo assim, pacientes tabagistas náo apresentaram maior risco de desenvolver erisipela, apesar disso, sabe-se que o tabagismo influencia na estase venosa e arteriosclerose dos vasos. Estudos apontam que o tabagismo é fator predisponente para outras doenças, como insuficiência venosa, que podem influenciar o curso da erisipela em adultos e idosos. ${ }^{(19)}$ Neste estudo, pacientes com insuficiência venosa tiveram o dobro de chances de desenvolver erisipela.

Estudiosos brasileiros ressaltam que, dentre os fatores clínicos causadores de erisipela, a obesidade é considerada fator de risco importante. ${ }^{(17)}$ Ao se abordar o fator obesidade, é interessante salientar que a doença comumente é associada a outros distúrbios, como hipertensão, insuficiência venosa, doenças cardíacas e diabetes, que também estão associados a erisipela. Em estudo na Suécia, as estimativas de efeito indicaram um risco quatro vezes maior de recorrência de recidiva para pessoas com obesidade grave. ${ }^{(1)}$ Esses dados divergem deste estu- do, no qual a obesidade, náo se revelou como fator de risco importante para a erisipela e recidiva tanto em adultos quanto em idosos.

Foi evidenciado que pacientes diabéticos não tiveram maior risco para desenvolvimento de recidiva, contrariando estudos na Suécia, em que a doença aumentou significativamente o curso complicado de erisipela. ${ }^{(1,13)}$ Além disso, nesse estudo, o linfedema náo se mostrou como fator de risco para recidiva de erisipela, embora nas demais pesquisas consideram essa patologia de importância clínica como fator de risco a ser considerado. ${ }^{(1,14,20)}$

A polifarmácia, que se caracteriza pelo uso de cinco ou mais medicaçóes de forma contínua, esteve presente em 31,1\% (73) dos casos, e não foi correlacionada com recidivas. ${ }^{(8)} \mathrm{O}$ grupo de idosos apresentou maior tendência a polifarmácia, o que pode ser justificado pois esta população geralmente mais comorbidades e ter desenvolvido mais fatores de risco para erisipela do que os jovens.

Quanto ao tratamento utilizado na primeira internação, pacientes que tiveram como antibioticoterapia a penicilina apresentaram risco sete vezes maior de recidiva. Segundo os guidelines internacionais, a penicilina $\mathrm{G}$ é o antibiótico de referência. ${ }^{(2)}$ Uma breve reflexão sobre as terapias farmacológicas e alguns fatores relevantes como a população e os hábitos de saúde, o uso indiscriminado de antibióticos como a penicilina de primeiro grupo, poderia estar associado a resistência, diminuindo assim seu efeito terapêutico, possibilitando a ocorrência de novos casos.

As ações do enfermeiro são de extrema importância no curso da doença, principalmente por meio da sistematização da assistência de enfermagem, e mais especificamente do processo de enfermagem, que possibilita ao enfermeiro determinar os diagnósticos de enfermagem e realizar as intervençóes adequadas para o cuidado e tratamento da erisipela. Ademais, torna-se necessário, durante as intervençóes no processo de enfermagem, o enfermeiro estar atento a questóes como: integridade da pele e risco de infecção, realizando inspeção visual e olfativa no curativo; queixas de dor do paciente, administrando analgesia; auxiliando na deambulação prejudicada, minimizando os riscos de queda; avaliando o 
preenchimento capilar; e melhorando a qualidade de vida, contribuindo, assim, de forma positiva no tratamento. ${ }^{(5)}$

A equipe de enfermagem necessita ter conhecimento para identificar os fatores de risco e fatores agravantes para erisipela através de anamnese realizada de forma correta. Além disso, necessita conhecer as possibilidades de tratamentos farmacológico e não farmacológico eficazes para atuar junto a equipe multidisciplinar, associando informaçôes pertinentes, para a resolutividade do caso e prevenção de recidivas.

Quanto ao papel da enfermagem na alta de todos os pacientes, sobretudo os que apresentam insuficiência venosa, o acompanhamento com consultas de retorno, a educação em enfermagem para o autocuidado com a pele e a mudança do estilo de vida como, repouso diário, cuidado com lesôes, identificação dos sinais de alerta, como os sinais flogísticos e prevenção de portas de entrada são cuidados de enfermagem importantes para evitar recidiva de erisipela.

Explicar ao paciente a necessidade de vigilância frequente quanto aos sinais do reaparecimento da doença se caracterizam por sintomas como dor no local, edema, vermelhidáo no membro afetado normalmente os membros inferiores, podendo também apresentar aumento da temperatura corporal, calafrios, êmese, náusea e mal estar, sendo imprescindível que este paciente procure atendimento especializado para início precoce do tratamento correto. ${ }^{(1)}$

No caso de pacientes com antibioticoterapia penicilina, em caso de recidiva da doença, o recomendado atualmente por diretrizes é a coleta de hemocultura para determinar a sensibilidade, a fim de guiar o tratamento de forma mais eficaz, pois estudo mostra que apenas 4,6\% de cepas são sensíveis à penicilina. Culturas de aspirados por agulha ou biópsias da pele inflamada ainda são pouco utilizadas e não representam efetividade comprovada. ${ }^{(13)}$

Como limitação, aponta-se dados que não foram possíveis de serem coletados, como a questão nutricional, tratamento pós alta, reinternações em outros hospitais, e a evolução da ferida após a internação, pois não constavam nos registros dos profissionais no prontuário informatizado. Além disso, não foi realizada a regressão logística, por isso os resultados estatísticos foram analisados com cautela.

\section{Conclusão}

Este estudo de coorte retrospectivo permitiu identificar associação entre fatores de risco e a ocorrência de casos de recidiva de erisipela em adultos e idosos. Os fatores de risco para a recidiva de erisipela com significância estatística foram: paciente apresentar insuficiência venosa e que tiveram como antibioticoterapia a penicilina. Com isso, torna-se importante identificar os fatores de risco associados a recidiva de erisipela, para que seja possível aprimorar à assistência aos pacientes com esse acometimento, bem como possibilitar cuidados de qualidade, com o intuito de diminuir os casos de recidivas e consequentemente as hospitalizaçóes constantes por erisipela.

\section{Colaborações}

Madeira ES, Figueredo LN, Pires BMFB, Souza SR, Souza PA contribuíram com a concepção, projeto, análise e interpretação dos dados, redação do artigo, revisão crítica relevante do conteúdo intelectual e aprovação final da versão a ser publicada.

\section{Referências}

1. Inghammar $\mathrm{M}$, Rasmussen $\mathrm{M}$, Linder $\mathrm{A}$. Recurrent erysipelas--risk factors and clinical presentation. BMC Infect Dis. 2014;14:270.

2. Stevens DL, Bisno AL, Chambers HF, Dellinger EP, Goldstein EJ, Gorbach SL, Hirschmann JV, Kaplan SL, Montoya JG, Wade JC; Infectious Diseases Society of America. Practice guidelines for the diagnosis and management of skin and soft tissue infections: 2014 update by the Infectious Diseases Society of America. Clin Infect Dis. 2014;59(2):e10-52. Erratum in: Clin Infect Dis. 2015;60(9):1448. Dosage error in article text.

3. Seybold U, Stubbe H, Draenert R, Bogner JR. Erysipel: Wann wird es kritisch? [Erysipelas]. MMW Fortschr Med. 2018;160(10):37-40. German. Review.

4. Campos MG, Souza AT, Vasconcelos JM, Lucena SA, Gomes SK. Feridas complexas e estomias: aspectos preventivos e manejo clínico. João Pessoa: Ideia; 2016.

5. Silva PL, Abreu GG, Fonseca JR, Souto SG, Gonçalves RP. Diagnósticos e intervenções de enfermagem em paciente com erisipela: estudo de caso em hospital de ensino. Rev Eletr Gestão Saúde. 2013:4(4):151226. 
6. Cruz RA, Miranda EG, Santos EC, Ferreira MG, Santana RA. Abordagem e reflexões para o cuidado do cliente com erisipela. Rev Bras Educ Saúde. 2016;6(1):22-6.

7. Hulley SB, Cummings SR, Browner WS, Grady DG, Newman TB. Delineando a pesquisa clínica. 4a ed. Porto Alegre: Artmed; 2015.

8. Secoli SR. Polifarmácia: interações e reações adversas no uso de medicamentos por idosos. Rev Bras Enferm. 2010;63(1):136-40.

9. Polit DF, Beck CT. Fundamentos de Pesquisa em Enfermagem. 7a ed. Porto Alegre: Artmed; 2011.

10. Miot HA. Tamanho da amostra em estudos clínicos e experimentais. J Vasc Bras. 2011;10(4):275-8.

11. Triola MF. Introdução à estatística, 10a ed. Rio de Janeiro: LTC; 2008.

12. James BR. Probabilidade: um curso em nível intermediário. 3a ed. Rio de Janeiro: IMPA; 2004.

13. Bläckberg A, Trell K, Rasmussen M. Erysipelas, a large retrospective study of aetiology and clinical presentation. BMC Infect Dis. 2015;15:402.
14. Pitché P, Diatta B, Faye O, Diané BF, SangaréA, Niamba P, et al. [Risk factors associated with leg erysipelas (cellulitis) in sub-Saharan Africa: a multicentre case-control study]. Ann Dermatol Venereol. 2015;142(11):633-8. French.

15. Santos RC, Menezes RM, Araujo GK, Marcolino EC, Xavier AG, Gonçalves RG, et al. Frailty syndrome and associated factors in the elderly in emergency care. Acta Paul Enferm. 2020;eAPE20190159.

16. Sočan K, Sočan M. Trends in the epidemiology of erysipelas in Slovenia. Acta Dermatovenerol Alp Pannonica Adriat. 2018;27(1):1-4.

17. Okajima RM, Freitas TH, Zaitz C. Erysipelas: a clinical study of 35 pacients hospitalized at the São Paulo Central Hospital of Irmandade da Santa Casa de Misericórdia. An Bras Dermatol. 2004;79(3):295-303.

18. Morais KC, Ferreira AC. 0 impacto da insuficiência venosa crônica no desempenho functional em mulheres. InterScientia. 2014;2(3):29-47.

19. Cruz RA, Miranda EG, Santos EC, Ferreira MG, Santana RA. Abordagem e reflexões para o cuidado do cliente com erisipela. REBES. 2016;6(1):22-6. Review.

20. Caetano M, Amorim I. Erisipela. Acta Med Port. 2005;18:385-94. 